\title{
Development of Raspberry-Pi Based IoT Agricultural System using Fuzzy Techniques
}

\author{
B. Sridhar, K. Lalitha Divya Sindhu, N. Ragasudha, M. Mounika, K. Premnath Chakri
}

\begin{abstract}
In India, agriculture plays an important role in national development. Agricultural problems have always hindered the country's development. The only solution to this is smart farming by modernizing traditional farming methods today. The Internet of Things (IOT) allows a variety of applications such as monitoring and selecting crop development, supporting irrigation decisions, etc. The result of this work is the monitoring of soil moisture, temperature, humidity, $\mathrm{pH}$, reproduction and water level . Field Using a sensor network that collects data from different types of sensors and then sends it to the main server using Raspberry pi, it uses vague logic in real time to make decisions based on the decisions made by the system.
\end{abstract}

Keywords: IOT, agricultural system, Fuzzy logic, Raspberry pi, Sensor nodes.

\section{INTRODUCTION}

$\mathrm{I}_{\mathrm{n}}$ ndia is a country of agriculture where more than $65 \%$ of the population depends on agriculture. Agriculture is the most important sector of the Indian economy. India's diverse climate guarantees the availability of all varieties of fresh fruits and vegetables. It ranks second in the world in the production of fruit and vegetables after China. The concept of quality is broad and covers various aspects, such as appearance, nutritional value, the presence of compounds related to health, safety and protection. On the other hand, freshness is strictly linked to the "age" of the product and, although it is an attribute linked to quality, these two concepts are different and nowadays it is no longer possible to " evaluate the quality of the product solely on the basis of its freshness.Providing safe, high quality food for a very complex and diverse market is a difficult task for the agricultural industry worldwide. Our biological systems are even more difficult in terms of long-term environmental sustainability and increased demand for products due to reduced resource base. Accelerating climate uncertainty due to global warming will exacerbate the problem. In order not to overcome these challenges,

Revised Manuscript Received on July 02, 2020.

* Correspondence Author

B. Sridhar*, Professor ECE department, LIET, Vizianagaram, India E-mail:srib105@gmail.com

K. Lalitha Divya Sindhu, Final year UG Students, ECE department LIET Vizianagaram, India.

N. Ragasudha, Final year UG Students, ECE department LIET Vizianagaram, India.

M. Mounika, Final year UG Students, ECE department LIET Vizianagaram, India.

K. Premnath Chakri, Final year UG Students, ECE department LIET Vizianagaram, India.

(C) The Authors. Published by Blue Eyes Intelligence Engineering and Sciences Publication (BEIESP). This is an open access article under the CC BY-NC-ND license (http://creativecommons.org/licenses/by-nc-nd/4.0/) it is necessary to apply improved methods derived from rich understanding. An aspect of this is the application and use of a variety of well-integrated technologies to provide more effective decision-making information.IOT promotes the creation of heterogeneous and distributed service ecosystems. These devices have access to the Internet for functions and data provided by built-in electronic devices, sensors and actuators, and physical devices equipped with software. There are many areas that use IOT technologies such as healthcare, smart home, industry, smart cities, agriculture, vehicles, smart buildings, retailers, factories, oil and gas. Everyone uses a different type of operating system. Scheduling the behavior of the operating system is currently limited to professional developers, as it requires the skills of different language bundles of actions, which may differ depending on the underlying material. A new approach that allows non-technical users to directly shape SO behavior can enhance the opportunities offered by the IOT.

Some recently offered tools support non-technical users, such as the so-called task automation (TA) tool .The quality of fruits and vegetables is important for consumers and is becoming a requirement for suppliers to provide food products with high quality standards. Consequently, in recent years, grading systems for fruits and vegetables have been put in place to meet the inspection needs of the food industry. In addition to this, the fruit and vegetable process involves several stages which can generally be classified into sorting, classification, packaging, transport and storage. Qualification is considered the most important steps towards the highest level of quality.

The promise of the agricultural IOT, which faces a huge challenge for global agricultural production, has almost doubled by 2050, is huge. Once fully developed, evaluated and implemented, it provides the opportunity to activate subdivisions and specific decisions in real time. Provides the ability to collect, protect and notify high-intensity data sets that can be filtered or processed through a computer field, either in the field or with the platform's high-performance computing system. It uses cloud algorithms, innovative algorithms, artificial intelligence and mechanical learning, huge data analysis and fast production proposals for farmers in almost real time. Many obstacles and challenges must be overcome before the agricultural IOT becomes a reality. From the lack of Internet connection and broadband connectivity in rural areas to the development of reliable detection devices and unavoidable algorithms for mechanical systems, low-cost IOT farming systems are capable of firing the right amount of energy at the right time and at the right time. Also, given the complexity and unknown elements associated with innovative technologies, including IOT agriculture, it is not surprising that Gartner's advertising campaign was followed.

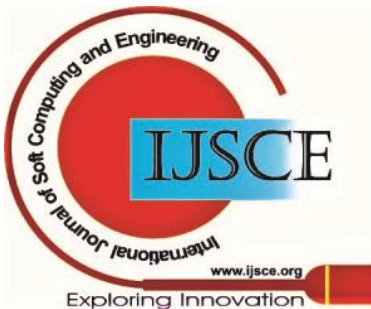




\section{Development of Raspberry-Pi Based IoT Agricultural System using Fuzzy Techniques}

Only time will tell if the agricultural IOT will be able to withstand Gartner's slow and difficult advertising cycle until it reaches the road to productivity. The proposed system presents a clever agricultural vision that can be used to collect important real-time data for use in a range of data processing and modeling approaches to create high quality and reliable decision-making information using connected sensors. . The system shows how data along a logical path contributes to the flow of convergence of information in the decision support system and how it can be converted into active steps. Much of the SMART vision comes with the idea that it can be firmly integrated into the round concept of smart, fast, technological, economic, political and cultural practices and systems. The book develops new ideas for the strong integration of ICT and IOT for the management of agricultural assets, arguing that the key to smart agriculture is to provide better financial performance and adopt technologies and methods in different environments in space and time. An innovative collaborative control protocol for robotics has also been introduced to help humans, robots and sensors perform harmonious agricultural tasks.

In a clear sense, fuzzy logic is an intelligent system of multi value logic. These values respond to uncertainty in most engineering applications by relating the degrees of certainty fuzzy logic for decision making. In this study, the system will be based on a fuzzy logic control. Fuzzy logic control is chosen for the following reasons:

I. It can be used in the design of systems where it is impossible to obtain its models for conventional control.

ii. It can be used to mimic the experienced human operator to model the control system.

iii. It is easily integrated into small microcontrollers and processors.

iv. Compared to conventional control, fuzzy logic is more precise.

This thesis work is carried out in the following objectives

1. Studying literature on Smart agricultural system and its applications in various IOT based techniques to get the three important attributes of automation ie., sensing the information from crop field, data processing, decision making.

2. Survey of the existing techniques for extracting the attributes of IOT .

3. Analysis and design of proposed system for detection.

4. Implementation of the proposed design by considering the data from various sensors.

5. Applying fuzzy based techniques of decision making to grade the levels and drive the output system on the basis of sensors data.

6. Display the current mode of operation of proposed system by using display devices such as LCD and monitors

In the section 2 presented a basic literature review, section 3 ,we discuss the smart Agricultural systems and its recent developments to implement this proposed system. We also discuss basics of IoT in detail which is the heart of the proposed system.

In the 4rd section, we discuss about the hardware details that are used in the proposed system. It includes Raspberry pi architectural details, mode of operation for this proposed system and block diagram and circuit diagram of the to a logical question. Most agricultural applications rely on

proposed system. In the section 5, we deal with the implementation of our proposed system that is "Quality check" is shown with various stages involved in the proposed system implementation. Detailed experiments and the results are presented in next section ie..5 with the output images along with their respective results along with the achievements of our proposed system and some possible future extensions of the system are proposed

\section{LITERATURE REVIEW}

Many researchers have conducted studies on the design of techniques to determine important parameters for assessing the quality of fruits and vegetables. S.Muthunpandian et al. [1] provided an automated system designed for continuous monitoring of crops. System Developing a system to improve water levels in the field by consuming energy in the field Useful for irrigation systems.

Joaquín Gutiérrez et al. [2] showed an automatic irrigation system that more effectively reduces water resources when we consider the period of water shortage. They found that they incorporated solar energy systems to minimize water consumption and reduce energy consumption. It was developed by a smartphone that takes data from Internet sensors. Mohan raj et al. [3] focuses on monitoring data in the agricultural cycle.

The system includes a GSM sensor powered by an ATMEL microcontroller and used to monitor changes in windmill temperature, at the water $\mathrm{pH}$ level. After that, when we think it's more convenient to monitor a large number of Raspberry Pi systems, IoT systems based on Aurdino are used.

Michael G. Williams [4] introduced a raspberry irrigation system. The Raspberry Pi-based system is used in various application for home automation, entertainment system, security. The development of the raspberry system is more interesting for the thrust environment [10].

\section{SMART AGRI SYSTEM}

According to a recent report by the Food and Agriculture Organization of the United Nations (FAO), the world's population will exceed 990 million by 2050 (FAO, 2009). Significant challenges must be overcome to achieve the level of agricultural productivity required to meet global demand for food, feed, fiber and fuel, which is proposed systemed by 2050. Agriculture has faced significant challenges in the past, but it must specifically target productivity. 2050 to address serious constraints, such as resource constraints, lack of skilled workforce, inaccessible land and climate change by 2050

Fine agriculture (PA) is presented as one of the many solutions to the great challenges facing agriculture and our world today. The Palestinian Authority has been using heterogeneity in space, time and production for 30 years to implement a simple approach, but it has been established as an effective management method that applies the right information at the right time. Write the correct amount of the AP "R" concept in time on the right (Khosla, 2010).

Over the years, PAs have evolved around the world and are slowly adopting new autonomous, disorganized and data-intensive technologies.

\section{Published By:}

Blue Eyes Intelligence Engineering

Retrieval Number: F3402039620/2020@BEIESP

DOI: 10.35940/ijsce.F3402.0710120

Journal Website: www.ijsce.org

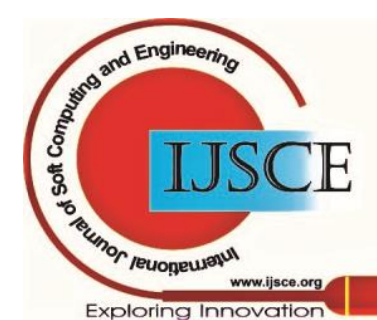


The first decade of the PA focused on the satellite land navigation service (GNSS) and its ability to detect and quantify spatial variability on the ground. For the past 20 years, we have focused on the automation of tractors and the development of technology for accurate input management, such as the nutrients of crops.

For more than 30 years now, location-based agricultural data collection has been exponentially developed through sensors and detection devices, setting a new example for management decisions based on evidence for precision management. Therefore, future agricultural practices, production success, efficiency and sustainability will largely depend on "data cultivation" as well as "land cultivation".

In addition, in order to overcome today's challenges, agriculture must become SMART and the IoT model (the IoT model that is evolving on the Internet of Everything (IoE)) represents the right architecture to restructure all areas and technologies related to smart. Agriculture. It can. To overcome the limitations of spatially diluted data, advances in proximity detection technology and data processing technology can provide information about soil, crops, and related environmental characteristics. Currently, the number of detection proximity techniques is increasing due to the advantages that non-invasive techniques are economical and economical. These sensors produce large amounts of data that must be collected, stored, communicated, processed, analyzed, merged, and interpreted to transform the data into new information and behavior (Figure 2.1).

Sensor data generated by a single sensor does not provide relevant information that can be used to fully understand the situation. Therefore, sensor data collected through multiple sensors must be merged, processed, and understood later. The integration of wireless sensors with agricultural applications for mobile and cloud platforms allows you to collect relevant information related to environmental conditions.

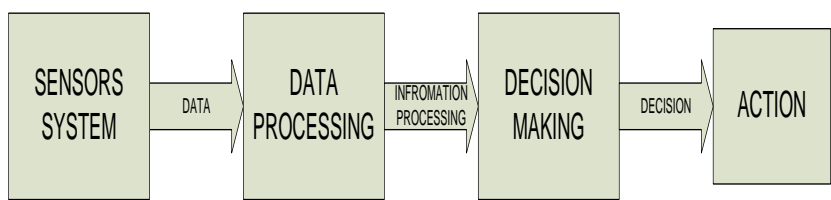

FIGURE .1 Agricultural internet of things model.

Temperature, rain, humidity, wind speed, pest infestation, soil humus content or nutrient fields can be used to make informed decisions to improve quality and quantity of production and minimize hazards and waste. Monitoring fields or crops based on applications also reduces the hassle of crop management in many locations. For example, farmers can now identify which areas are fertilized (or incorrectly incorrect) or need to be irrigated and assess the impact of practices on future yields.

The IoT agricultural model can be designed as shown in Figure 1.

Another key to this model is that the "decision making" process uses a dynamic model that evolves over time as experience accumulates. This allows for the development of agricultural knowledge based on empirical data collected to update best practices.

\section{HARDWARE DESCRIPTION}

\section{A. RASPBERRY PI 4 - MODEL B}

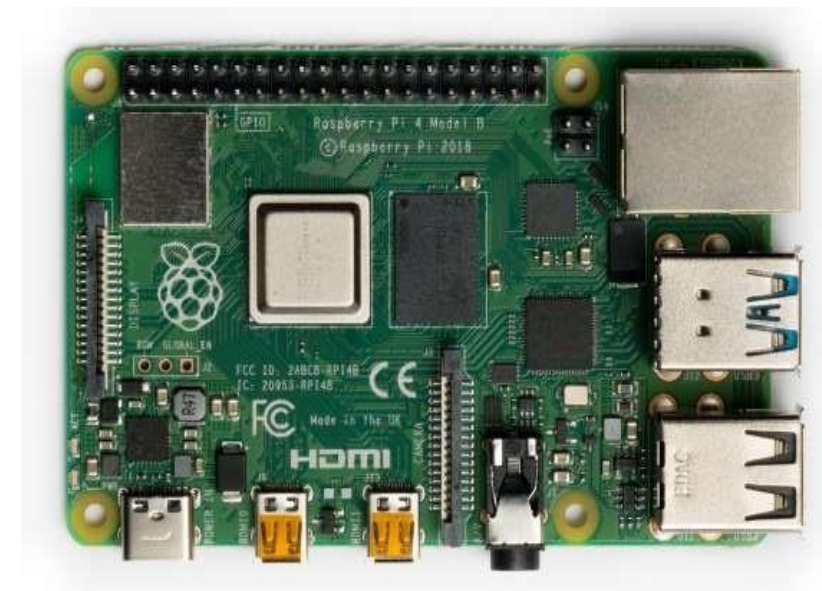

Fig 2. Raspberry pi 4 model b

The Raspberry Pi 4 Model B (Pi4B) is the next-generation Raspberry $\mathrm{Pi}$ to support more RAM and significantly improves CPU, GPU and I / O performance. Similar in form factor, power and cost folder, similar to Raspberry Pi 3B + previous generation. Pi4B can be used with 1, 2 or 4 gigabyte LPDDR4 SDRAM.

The fig:2 Raspberry pi 4 model. The Raspberry Pi 4 Model B was released in June 2019 with two ARG Cortex-A72 $1.5 \mathrm{GHz}$ 64-bit quad-core processors, built-in Wi-Fi 802.11ac, Bluetooth 5, full Gigabit (GB) Ethernet (no performance limit) and two. It supports USB 2.0 ports, two USB 3.0 ports and dual screens via a pair of micro HDMI ports (HDMI Type D) up to $4 \mathrm{~K}$ resolution. The $\mathrm{Pi} 4$ is powered by a USB-C port, so it can provide extra power to peripherals when used with a suitable PSU.

\section{B. BENCHMARKS \& PERFORMANCE IMPROVEMENTS}

Raspberry Pi is the latest addition to the Raspberry Pi brand in the computer category. Processor speed has been significantly improved depending on multimedia performance and connectivity. These improvements are noticeable compared to the previous generation Raspberry $\mathrm{Pi}$ 3 Model B +.

However, backward compatibility is maintained and energy consumption is maintained. In terms of end use, the performance of the new Raspberry Pi 4 Model B is the same as that of an x86 entry-level computer. Among the key features of this latest Raspberry Pi (fig:3) computer are the following:

- A high-performance 64-bit quad-core processor.

- Support for two displays with resolutions up to $4 \mathrm{~K}$ via a pair of micro-HDMI ports

- Hardware video decoding up to 4Kp60

- 4 GB of RAM

- Connection to the 2.4 / 5.0 GHz dual-band wireless local area network

- Features of Bluetooth 5.0 / Gigabit Ethernet / USB 3.0 / POE (via a separate POE HAT additional module) SPECIFICATIONS

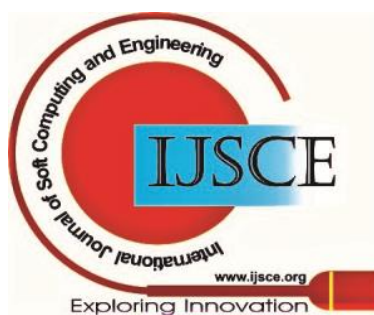




\section{Development of Raspberry-Pi Based IoT Agricultural System using Fuzzy Techniques}

- Processor: Broadcom BCM2711, quad-core Cortex-A72 (ARM v8) SOC 64 bits @

$1.5 \mathrm{GHz}$

- Memory: 1 GB, 2 GB or 4 GB LPDDR4 (depending on the model)

- Connectivity: $2.4 \mathrm{GHz}$ and $5.0 \mathrm{GHz}$ wireless LAN IEEE 802.11b / g / n / ac, Bluetooth 5.0, BLE Gigabit Ethernet 2 × USB 3.0 ports $2 \times$ USB 2.0 ports.

- GPIO: standard 40-pin GPIO header (fully backward compatible)

- Video and sound: 2 micro HDMI ports (up to 4Kp60 compatible) 2-way MIPI DSI display port 2-way MIPI CSI camera port 4-pole stereo audio port and composite video port

- Multimedia: H.265 (4Kp60 decoding); H.264 (1080p60 decoding, 1080p30 encoding);

OpenGL ES graphics card support, SD 3.0: Micro SD card slot for operating system loading and data storage

- Input power: $5 \mathrm{~V}$ DC via USB-C connector (minimum 3A1) 5 V DC via GPIO header

(3A1 minimum) Power over Ethernet (POE) activated (requires a separate POE HAT)

- Environment: operating temperature $0-50^{\circ} \mathrm{C}$

- Lifespan: the Raspberry Pi 4 Model B will remain in production until at least January

2026.

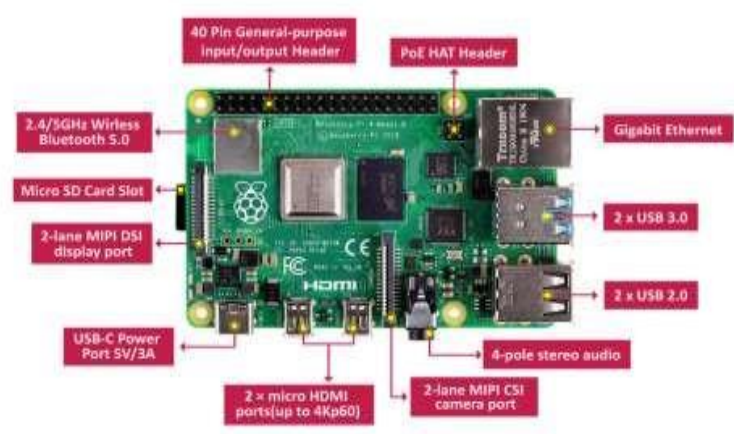

FIG 3 Diagram of Rasberry pi4

\section{PROPOSED METHOD}

\section{A. BLOCK DIAGRAM DESCRIPTION}

The block diagram of the proposed system is shown in Figure 4. The material elements are connected as shown. During working time, you must initialize the system and check the hardware connection. Raspberry-pi ensures that all connections are connected. In this proposed system, we will connect two sensors 1) temperature sensor 2) soil moisture sensor. In addition, to develop a more accurate system, historical data on irrigation volumes used in previous periods are considered to adjust the amount of water required for irrigation. The circuit diagram is shown in fig:5

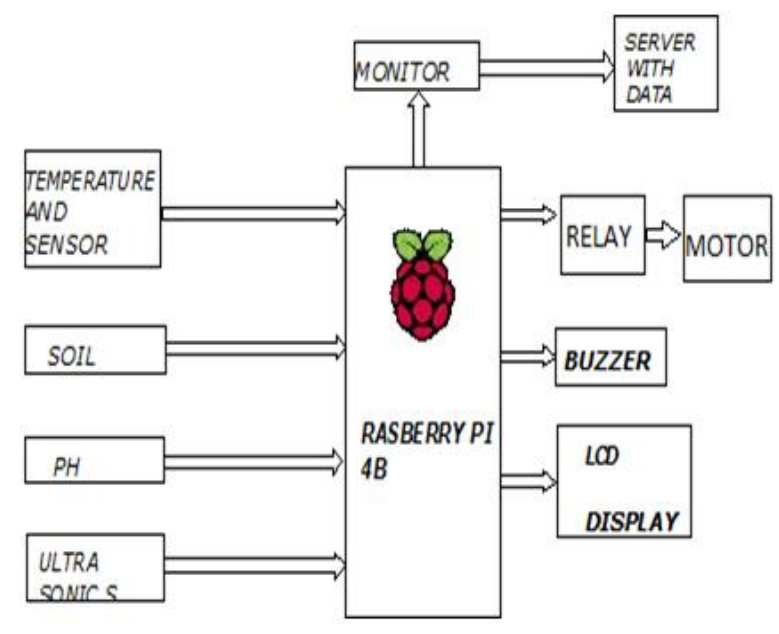

FIG 4. Block diagram of IOT Based automatic crop field monitoring
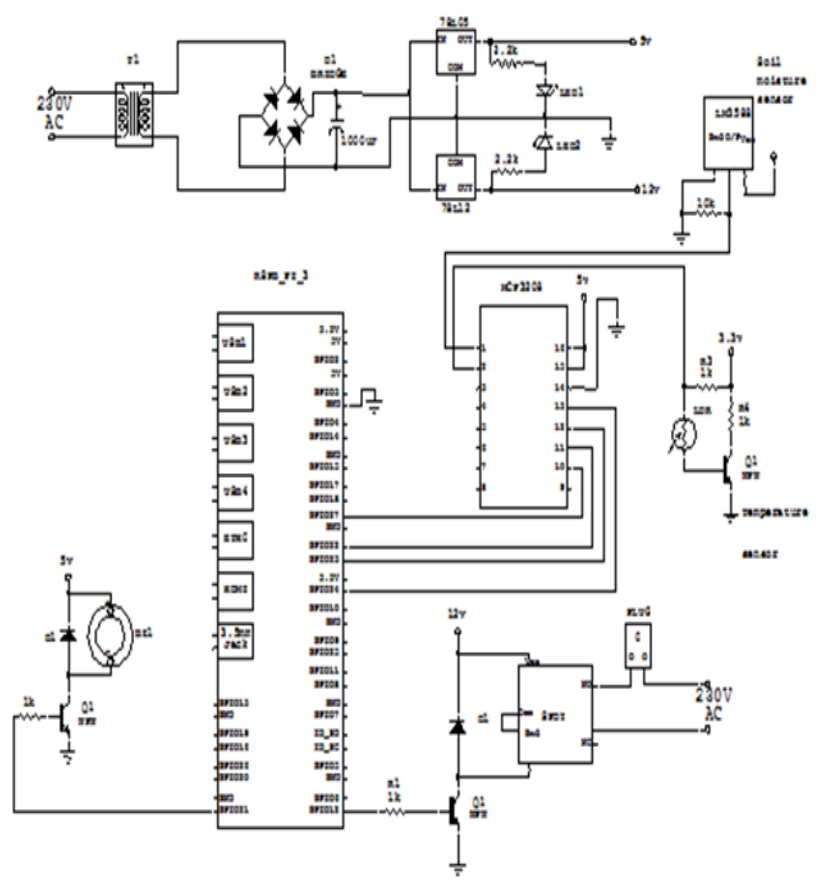

FIG 5:Circuit Diagram

These two sensors are placed in one cut and data is collected from these sensors in the form of analog values, so the analog values correspond to IC3208, which converts the analog values to 12-bit digital values. With Raspberry-pi, which sends data to the database using $\mathrm{Wi}-\mathrm{Fi}$, the sensor is calibrated so that the minimum humidity conditions are measured in the $2.4 \mathrm{v}$ field. The threshold trend varies depending on the season and the cultural field.

At this $2.4 \mathrm{~V}$, the Raspberry Pi activated the relay according to this threshold voltage and the relay is compared to $2.4 \mathrm{~V}$ when the value is less than 2.4 when the date comes from the controller. When the field is dry, a signal is sent to the engine running when the value is greater than 2.4 volts when the field is wet. Temperature change is calculated using different temperature sensors taking into account the correction values and the data from these sensors are correlated at all times and the exact level of soil drying is measured.

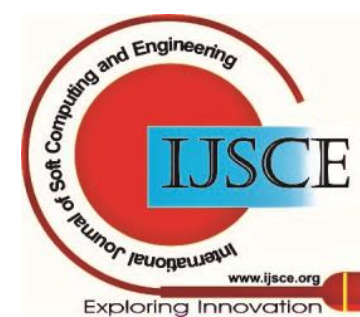


To automatically turn off the signal sent to the engine, an audible signal indicates that the situation changes when the engine is "turned on" and "turned on". Python programming is selected to implement the algorithm proposed by LINUX. Operating system

\section{B. Python libraries}

Python is an open source high level programming language. For its variety of libraries and simplicity, Python has become their applications. In this research study, Python was used to write a program that generated plots of live streaming sensor data. Important libraries needed for

development of this program that were downloaded from Python organization [3] are

as listed below.

i. Numpy library

ii. Drawnow library iii. Serialpy library iv. Matplotlib library

To install these libraries using the windows command terminal, PIP which is a program for installing Python libraries was first installed in Python directory. In order to view sensor data plots, the user had to connect the central station to the USB port of the personal computer and then opened Python code with "VDLE for Python (Python integrated development environment)”.

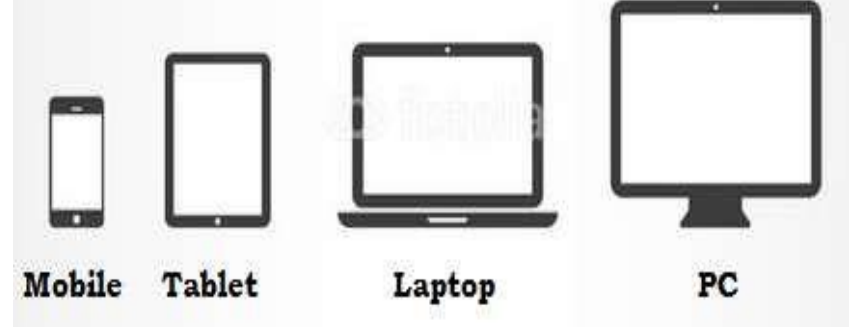

Fig: 6 Electronic display device

Hardware requirement for implementation of the function module

- Raspberry-PI 4 model B

- DHT22 sensor (Humidity and Temperature sensor)

- Soil moisture sensor

- Ultrasonic sensor

- Ph sensor

- Relay

- Buzzer

- Power Supply Unit.

- Miscellaneous Components.

Note: Based On Requirement of Application uses Ethernet (or) WI-FI (or) 3GDongle.

Software requirement for implementation of the function module

For Raspberry Pi:

- A Wheezy Raspbian Installed System Required Python

- The Qt Creator is complete GUI Application development framework for

Raspberry-Pi. Language:

- All editions provide a complete Embedded C/ C++/Python high level programming language that many fields use for

development environment includes extensive middleware libraries.

- Raspbian (Linux Oriented Recommended).

\section{FUZZY TECHNIQUES IMPLEMENTATION}

Fuzzy logic has been used for a wide variety of applications. Among these applications, it has been used for irrigation control to achieve the intelligent decision making of a human operator. It determines when the irrigation should begin based upon the information from soil sensors. It can also learn the irrigation schedules by itself during on-line control process [2]. Literature points out that there are a lot of papers in which fuzzy logic has been applied. For example, Nandwana et al [3] used fuzzy logic control on a solar powered pump for irrigation of green vegetable plants. Others such as Wang et al [3] investigated the use of fuzzy logic in the lumber drying process. In the investigation, a conventional adaptive controller was implemented as well, and its performance was compared with that of fuzzy logic adaptive controller. The results were outstanding for fuzzy logic controller as it managed to overcome complex, nonlinear, and time variant nature of drying process when conventional control was used. Human intervention in agricultural processes has so far been minimized by existing control systems. Generally, in such processes fuzzy logic has been implemented in the design of such intelligent systems and is renowned for its ability to lessen the complexity of certain systems without using mathematical models. This input variable had three membership functions named "dry " , “damp" and "wet" membership functions. The output variable (see Figure:7 itself was named "sprinkler" and had two membership functions named "sprinkler on" and "sprinkler off.

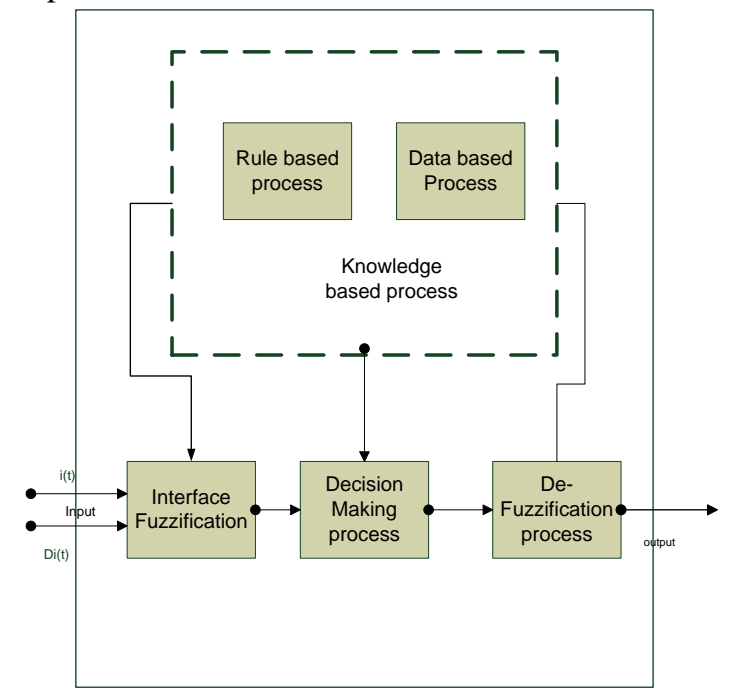

Figure 7. The block diagram of fuzzy logic controller

\section{Fuzzy logic results of soil moisture content}

To detect the degree of soil moisture content, the input variable named soil moisture was used in this study (see Figure 8. The maximum analog steps available for this input variable were 1000 steps.

Blue Eyes Intelligence Engineering and Sciences Publication

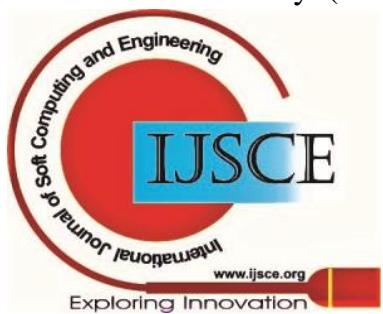




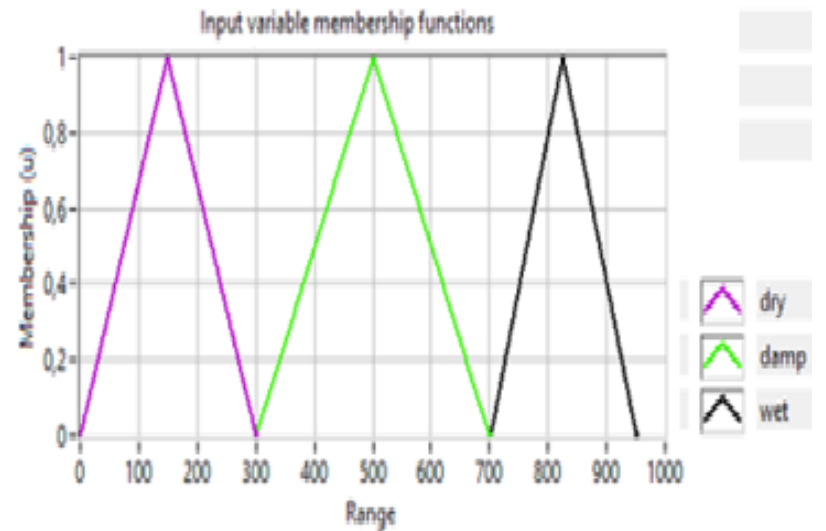

Figure 8: Soil moisture input variable with dry, damp and wet membership functions

The output variable of the soil moisture content is shown in Figure 8. The fuzzy inference rules for soil moisture sensor input and its output variable were as shown below.

1. If SOIL MOISTURE is DRY, then Motor on.

2. If SOIL MOISTURE is DAMP, then Motor on OFF.

3. If SOIL MOISTURE is WET, Motor on OFF. Fuzzy logic results of temperature and humidity

In fuzzy logic the input variable is further classified into what is known as membership functions. Membership function can be seen as part of the input variable with a certain range limiting it within the chosen values of the available input variable membership function of the temperature input variable. The orange triangle in Figure 4.5 represents a " normal " membership function of the temperature input variable with temperatures ranging from $20 \mathrm{C}$ to $32.5 \mathrm{C}$ while the red trapezoid represents a "hot" membership function of temperatures from $27 \mathrm{C}$ to $100 \mathrm{C}$. The degree of membership is represented on the y axis of Figure 9. In " cold " membership function for example, the degree of membership (y axis value) was higher when temperature was $17 \mathrm{C}$ than when it was $10 \mathrm{C}$.The knowledge of the degree of membership in a membership function was not important in this study as

the system designed was not a system where accuracy was a matter of concern as it would be design of medical systems for instance.

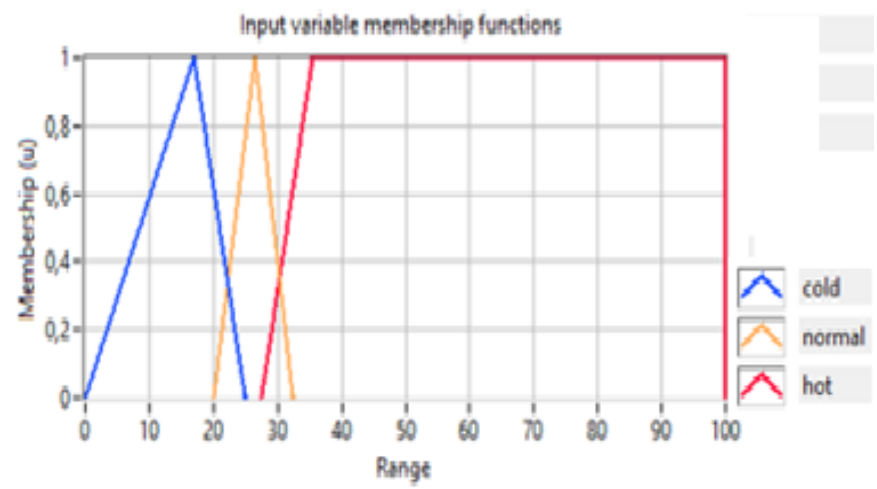

Figure 9: Temperature input variable with cold, normal and hot membership functions membership function of the temperature input variable. The orange triangle in

Figure 4..16 represents a “ normal ” membership function of the temperature input variable with temperatures ranging from $20 \mathrm{C}$ to $32.5 \mathrm{C}$ while the red trapezoid represents a "hot" membership function of temperatures from $27 \mathrm{C}$ to $100 \mathrm{C}$. The degree of membership is represented on the y axis of Figure 4..16. In " cold " membership function for example, the degree of membership ( $\mathrm{y}$ axis value) was higher when temperature was $17 \mathrm{C}$ than when it was 10 C.The knowledge of thedegree of membership in a membership function was not important in this study as the system designed was not a system where accuracy was a matter of concern as it would be design of medical systems for instance.

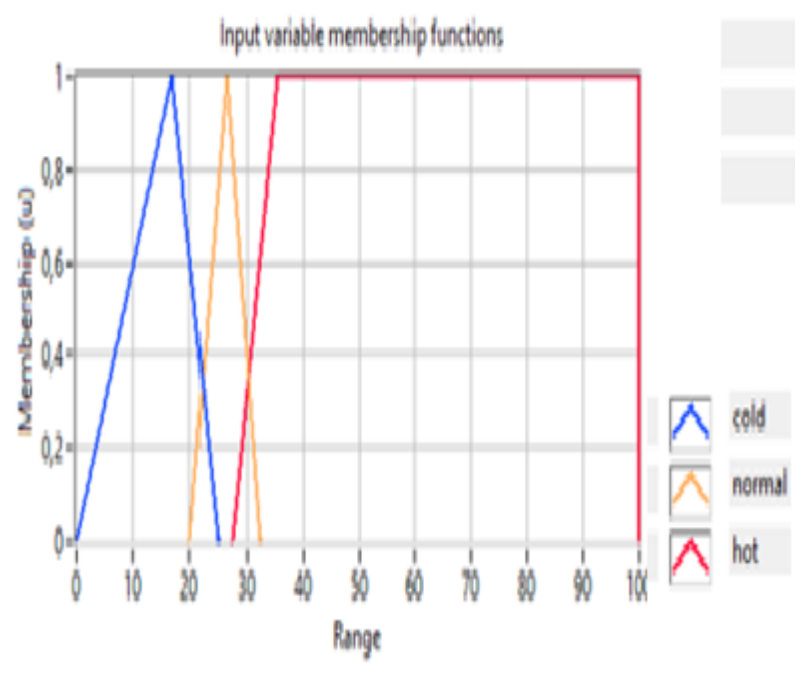

Figure 10: Temperature input variable with cold, normal and hot membership functions

Figure 10 shows the second input variable of the system which is humidity. This humidity had its values expressed as percentage on the $\mathrm{x}$-axis. The maximum value of humidity the system could detect was $100 \%$. sensors was realized as a value belonging to a particular membership function of the humidity input variable. Any value of humidity within the range of 0 to $75 \%$ undoubtedly belonged to " low " humidity membership function. This group of values that represented low humidity was known as "low" humidity membership function. $75 \%$ to $85 \%$ humidity range represented "optimal”

humidity membership function while $85 \%$ to $100 \%$ range represented "high" humidity membership function. In this study the whole concept of how these inputs were viewed in fuzzy logic sense is shown below:

Temperature input variable $=$ ( " cold " membership function + "normal" membership function + "hot" membership function).

In binary logic (also known as traditional logic) the input could be classified only into two states of either high or low. This was different in fuzzy logic because the input could be classified into many states depending on the system designer 's choice. A simple example is that of a person when his height is to be judged. His height could be classified only as either tall or short in binary logic but in fuzzy logic the same person' s height could be classified as short, medium short, tall, medium tall and so forth. Humidity decision as shown in fig:11

\section{Published By:}

Blue Eyes Intelligence Engineering

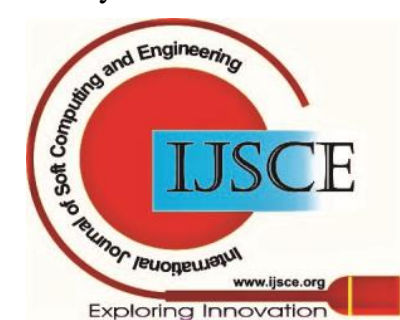


Humidity input variable $=$ ( "low "membership function + “optimal” membership function + "high" membership function)

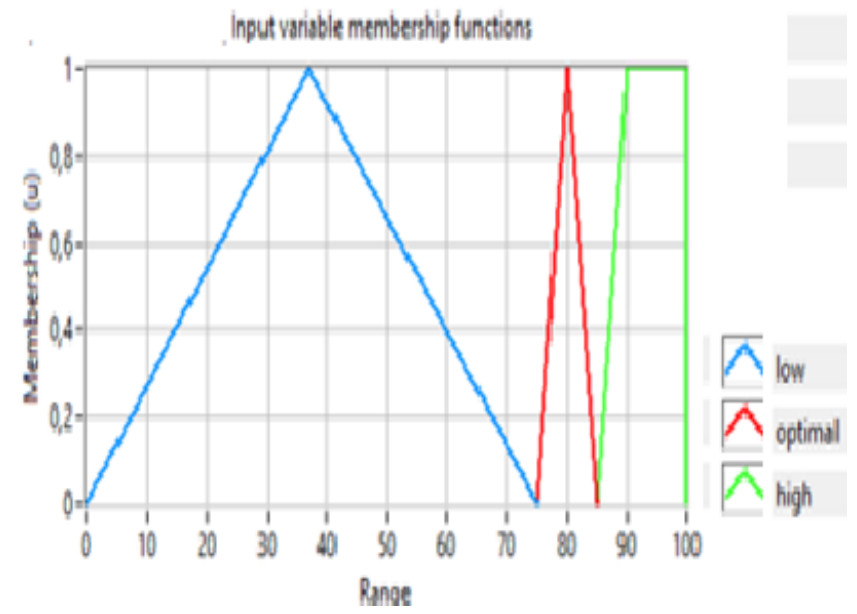

Figure:11 Humidity input variable with low, optimal and high membership functions

The designed control rules of temperature and humidity are shown below:

If TEMPERATURE is HOT and HUMIDITY is HIGH, then OUTPUT is Raspberry-pi ON AND

MOTOR OFF

If TEMPERATURE is HOT and HUMIDITY is OPTIMAL, then OUTPUT is Raspberry-pi ON AND MOTOR ON

If TEMPERATURE is HOT and HUMIDITY is LOW, then OUTPUT is Raspberry-pi ON AND

MOTOR ON

If TEMPERATURE is NORMAL and HUMIDITY is LOW, then OUTPUT is Raspberry-pi ON

\section{AND MOTOR ON ELSE MOTOR OFF}

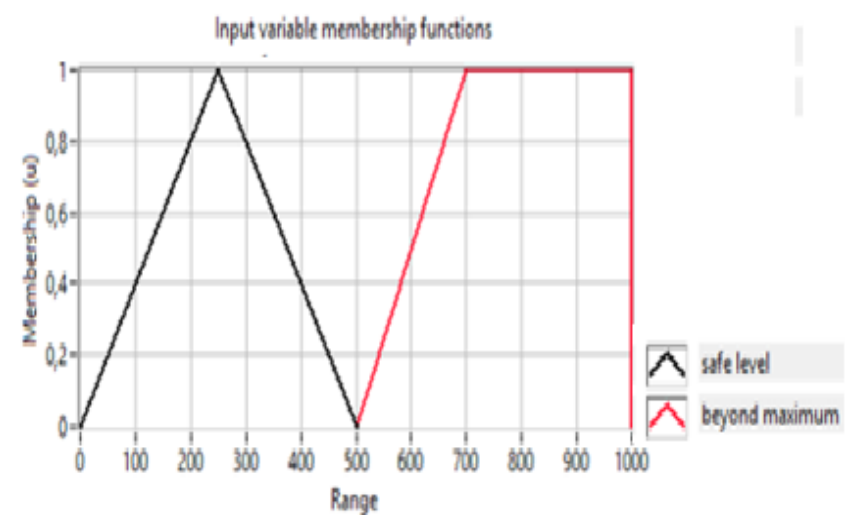

Figure:12 Ultrasonic sensor input variable with " safe level" and "beyond maximum" membership functions.

The Ultrasonic sensor input variable is represented as analog steps on the $\mathrm{x}$ axis of Figure. The Raspberry pi has 1023 steps of Analog readings through its inputs. The system only needed 1000 steps to represent Pest effects readings as inputs to sensor of which a range of 0 to 500 analog steps were readings representing a "safe level" membership function. The red trapezoid represented a " beyond

\section{Fuzzy logic results of Ultrasonic sensor}

maximum " membership function with a range of 501 to 1000 Analog steps. The output variable “Alaram On/OFF had two membership functions. These output membership functions were used as destination of fuzzy control rules designed to control Pest effects concentration. The designed fuzzy logic rules to control Pest effects concentration were as follows:

1. If Ultrasonic sensor is SAFE LEVEL, then Alarm is OFF and No send the message to former about the crop suffered by some pest

2. If Ultrasonic sensor is BEYOND MAXIMUM, then Alarm is On and send the message to former about the crop suffered by some pest

\section{Fuzzy logic results of $\mathbf{P H}$ sensor}

The PH sensor input variable is represented as analog steps on the $\mathrm{x}$ axis of Figure. The Raspberry pi has 1023 steps of analog readings through its inputs that represents $\mathrm{PH}$ value 0 to 14$)$.. The system only needed 1000 steps to represent $\mathrm{PH}$ readings as inputs to sensor of which a range of 0 to 500 analog steps were readings representing a " acid level " membership function. The red trapezoid represented a " base level" membership function with a range of 501 to 1000 analog steps. The output variable “ soil acid/basic property then suggestion to be given to former use fertilizers with respective crops " had two membership functions. These output membership functions were used as destination of fuzzy control rules designed to control Pest effects concentration. The designed fuzzy logic rules to control PH effects concentration were as follows:

1.If $\mathrm{PH}$ sensor is Acid level, then THEN SMS to be sent use fertilizers with respective crops to neutralize l

2.If $\mathrm{PH}$ sensor is basic level, then SMS to be sent use fertilizers with respective crops to neutralize l

In order to implement the above fuzzy logic the entire fuzzy function are include the single flow chart as shown in the fig: 13

\section{Published By:}

Blue Eyes Intelligence Engineering

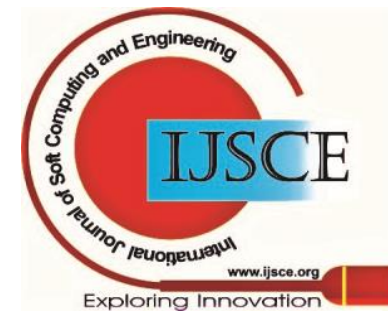




\section{Development of Raspberry-Pi Based IoT Agricultural System using Fuzzy Techniques}

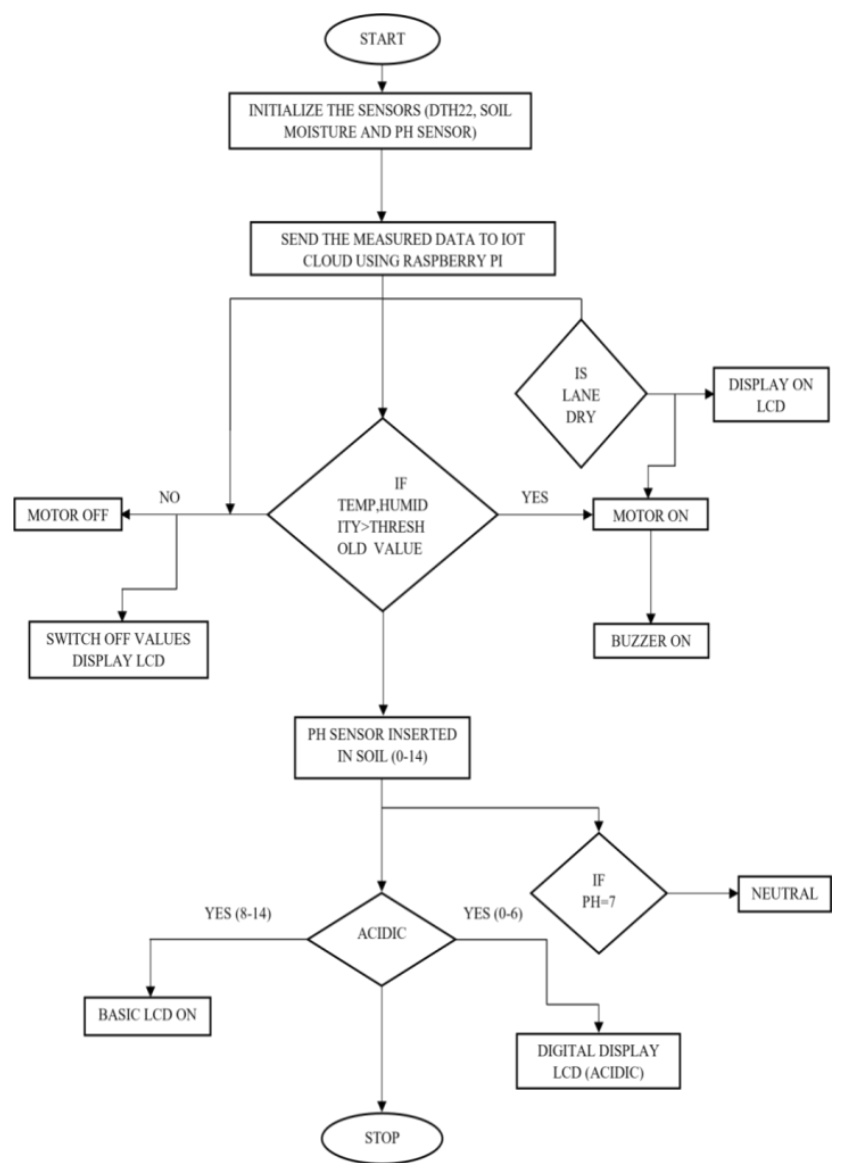

Fig:13 Flowchart of the program

Procedure to implement the proposed system:

The entire working of the proposed system takes place in an efficient sequential manner as follows.

1. The sensors present in the various parts of the fields are connected to the Raspberry pi.

2. The Raspberry pi received the data periodically after some interval of time.

3. The data received by Raspberry pi serially.

4. The received data is then stored in the pi database as well as it is sent as to the user.

5. The data is also uploaded to the thinkspeak application programming interface.

6. The data sent to thinkspeak can be viewed in the graphical form in the channel

7. The watering of field is based upon the level set for the moisture. 9. Based on the fuzzy logic sensors such as temperature, moisture and humidity the motor is turned on and when the desired level is reached it' s turned off.

8. And a Ultrasonic' s sensor is placed nearer to the plants, if any pest will effected an automatic information is given to formers and alarm on. $\mathrm{w}$

$9 \mathrm{PH}$ sensor is placed in soil such that checks the soil property continuous 24x7 which is displayed in live in Raspberry Pi's IP address and alerts messages will be sent to former .

The Results of the proposed method is discussed in the next section

\section{RESULT AND DISCUSSION}

Original material kit designed as shown. The results are real-time, as shown in Figure 13. The temperature and

humidity of the soil are also shown. The data received from the sensor is stored in the cloud and the farmer can monitor it via mobile device / computer. Farmers actually observe the automatically due to intervention in the crop sector.

The microcontroller has always processed and correlates huge data obtained from the limit sensor. Here, the system displays temperature and soil moisture values based on two engine status sensors. The state of the system can be ntrolled from a remote location and the complexity of the system is reduced, making it easy to deal with hardware problems.

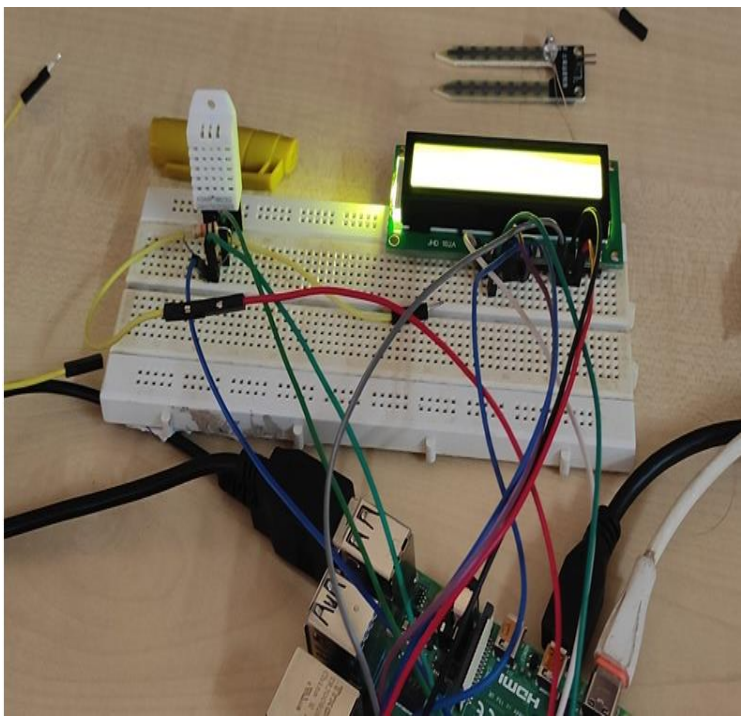

Fig: 13 photograph of proposed system hardware.

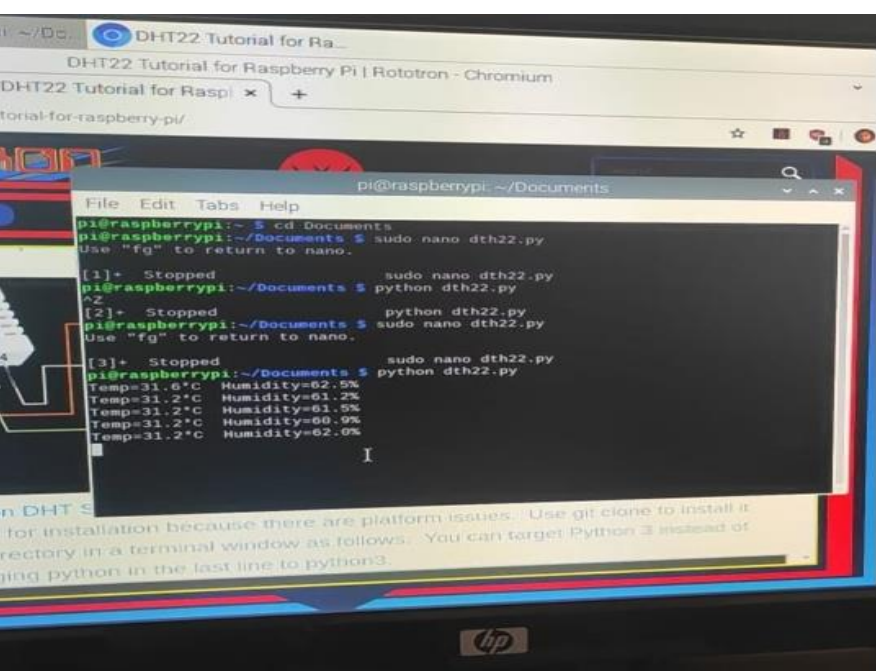

Fig: 14 photograph of proposed system outputs of the proposed system.

\section{CONCLUSIONS}

This intelligent farm monitoring system is a very important system as it supports the farmer through an automated system with automated detection techniques. The farmer can obtain information on the agricultural field, because the humidity, the temperature and the moisture content of the soil sensors are used efficiently in the circuit to bring the calibrated information to the system.

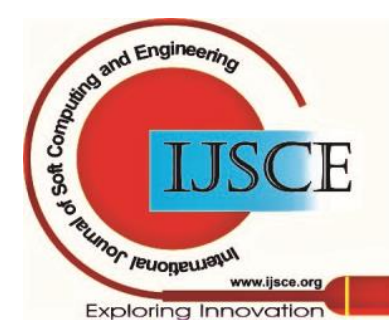


Five Raspberry pi sensors and microcontrollers from the three nodes successfully connect to multiple nodes. Decision making can be done by processing data from all sensors using fuzzy logic techniques. The outputs of the proposed system are on / off of the engine for water irrigation, sending information to trainers and monitoring through the cloud. All observational and experimental experiments show that the proposition is a perfect solution for field activity, irrigation problems. Applying these systems to the sector can certainly help improve crop yields and overall yields. . With this approach, the agricultural system

Automation also provides real-time information about land and crops to help farmers make the right decisions. Cloud computing is a "new style of computer that provides dynamically expandable and often virtualized resources as Internet services." Sensor is used to control the irrigation system so that you can easily perform troubleshooting when needed. Here, the proposed algorithm based on correlation data reduces the complexity of the material compared to other proposed systems. The threshold voltage is selected for sensor calibration, taking into account the last month of soil temperature and humidity values. The thresholds may vary depending on the crop and the farm. In the future, you will introduce machine learning algorithms that will be used to process data and reduce the complexity of the material. The material resources of the agricultural information network are integrated into the resource group using activation technology, allowing dynamic distribution and balancing of resource loads, significantly improving efficiency.

\section{ACKNOWLEDGMENT}

We would like to say thanks our Institution Management, Principal, Head of the Departments for they support in terms provided IoT Lab facility and constant encouragement to finish the work

\section{REFERENCES}

1. R .Nageswara Rao, B. Sridhar. "IoT based smart crop-field monitoring and automation irrigation system" , 2018 2nd International Conference on Inventive Systems and Control (ICISC), 2018

2. Lucio Colizzi, Danilo Caivano, Carmelo Ardito, Giuseppe Desolda et al. "Introduction to agricultural IoT" , Elsevier BV, 2020

3. Malusi Sibiya, Mbuyu Sumbwanyambe "Embedded Fuzzy Logic Microcontroller For Plant Condition Monitoring: A Wireless Sensor Network Odyssey" , 2019 Southern African Universities Power Engineering Conference/Robotics and Mechatronics/Pattern Recognition Association of South Africa (SAUPEC/RobMech/PRASA), 2019

4. Quanxing Zhang, Chwan-Hwa Wu, K. Tilt. "Application of fuzzy logic in an irrigation control system" , Proceedings of the IEEE International Conference on Industrial Technology (ICIT'96), 1996

5. Ulfah Putri Bisba, Edi Mulyana, M. Ali Ramdhani, Mohamad Irfan. "The Implementation of The Fuzzy Sugeno Algorithm On an IoT-Based Temperature and Humidity Monitoring System" , 2019 IEEE 5th International Conference on Wireless and Telematics (ICWT), 2019

6. Philip A. Adewuyi. "Performance Evaluation of Mamdani-type and Sugeno-type Fuzzy Inference System Based Controllers for Computer Fan" , International Journal of Information Technology and Computer Science, 2012

\section{AUTHORS PROFILE}

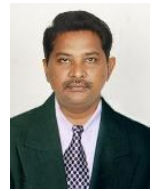

Dr. B.Sridhar obtained the Ph.D degree in medical image processing from theJNT University Kakinada in the year 2015. He has joined the Electronics and communication Engineering Department at Lendi Institute of Engineering and Technology in 2011 and continues to work as Professor. His research interests are Medical Image Processing, Machine Learning and nano VLSI design. He is a life member from IEEE,IETE,IE.He has a strong basic knowledge on image processing techniques using advanced and hybrid methods in both spatial and frequency domain. He also have a very good knowledge on neural networks and deep learning methods, Good programming skills using MATLAB,PHYTON applied on medical image data sets. He has published 29 research papers in, National, International Journals so far

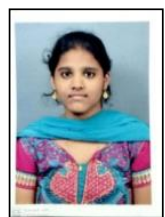

K. Lalitha Divya Sindhu an under graduate student in the department of Electronic and Communication engineering at Lendi Institute of Engineering and Technology, Vizianagaram India. I am also fond of doing the projects on IOT based.Also I am the member of Institute of engineers

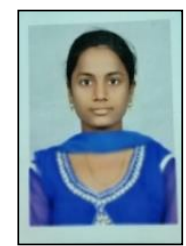

N. Raga Sudha an under graduate student in the department of electronic and communication engineering at Lendi Institute of Engineering and Technology, Vizianagaram. I am also fond of doing the projects on IOT based .I have a very good programming skills like java Python, etc.

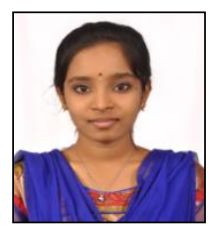

M. Mounika an under graduate student in the department of electronic and communication engineering at Lendi Institute of Engineering and Technology, Vizianagaram. I am also fond of doing the projects on IOT based. Also persue the skills like coding, designing and so on.

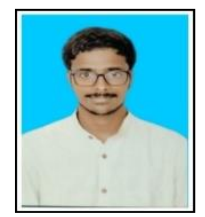

K. Premnath Chakri an under graduate student in the department of electronic and communication engineering atLendi Institute of Engineering and Technology, Vizianagaram. I am also fond of doing the projects on IOT based. Also persue the skills like coding, designing and so on.
Published By:

Blue Eyes Intelligence Engineering
(C) Copyright: All rights reserved. and Sciences Publication

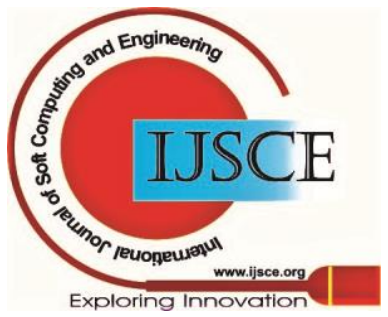

